Commun. Korean Math. Soc. 19 (2004), No. 4, pp. 765-773

\title{
STRONG LAWS OF LARGE NUMBERS FOR ASYMPTOTICALLY QUADRANT INDEPENDENT RANDOM FIELDS
}

\author{
Mi-Hwa Ko, Tae-Sung Kim, and Hyun-Chull Kim
}

ABSTRACT. In this paper we define the notion of asymptotically quadrant independent random field and derive the strong laws of large numbers for this random field.

\section{Introduction}

Let $d$ be a positive integer, $\mathbb{R}^{d}$ the $d$-dimensional Euclidean space equipped with the coordinate-wise partial order $\leq$ and $\mathbb{Z}_{+}^{d} \subset \mathbb{R}^{d}$ the $d$ dimensional lattice. The notation $\underline{m} \leq \underline{n}$, where $\underline{m}=\left(m_{1}, m_{2}, \cdots, m_{d}\right)$ and $\underline{n}=\left(n_{1}, n_{2}, \cdots, n_{d}\right)$, thus means that $m_{k} \leq n_{k}$, for $k=1,2, \cdots, d$. We also use $|\underline{n}|$ for $n_{1} \times n_{2} \times \cdots \times n_{d}, \underline{n} \longrightarrow \infty$ is to be interpreted as $n_{k} \longrightarrow \infty$, for $k=1,2, \cdots, d$ and $\|\underline{n}\|$ for $\max _{1 \leq k \leq n}\left|n_{k}\right|$.

Let $\left\{X_{\underline{j}}: \underline{j} \in \mathbb{Z}_{+}^{d}\right\}$ be a field of $d$-dimensional random variables on some probability space $(\Omega, K, P)$ with $E X_{\underline{j}}=0$. The field $\left\{X_{\underline{j}}: \underline{j} \in \mathbb{Z}_{+}^{d}\right\}$ fulfills the strong law of large number if as $\underline{n} \longrightarrow \infty$,

$$
|\underline{n}|^{-1} \sum_{\underline{1} \leqslant \underline{j} \leqslant \underline{n}} X_{\underline{j}} \longrightarrow 0 \text { a.s. }
$$

where $\underline{n}=\left(n_{1}, n_{2}, \cdots, n_{d}\right)$ and $|\underline{n}|=n_{1} \times n_{2} \times \cdots \times n_{d}$.

The purpose of the paper is to establish the strong laws of large numbers for the fields of $d$-dimensional random variables which are not necessarily independent. Evidently, we have to impose certain restrictions on the fields, that means we require the weak dependence. A field

Received July 19, 2003.

2000 Mathematics Subject Classification: 60E15, 60F15.

Key words and phrases: strong law of large numbers, pairwise positive quadrant dependent random variables, asymptotically quadrant independence, random fields. 
$\left\{X_{\underline{j}}: \underline{j} \in \mathbb{Z}_{+}^{d}\right\}$ is said to be pairwise positive quadrant dependent (PQD) if for every $\underline{i}$ and $\underline{j}$, and for every real $s, t$,

$$
P\left\{X_{\underline{i}}>s, X_{\underline{j}}>t\right\}-P\left\{X_{\underline{i}}>s\right\} P\left\{X_{\underline{j}}>t\right\} \geq 0
$$

or, as an equivalent condition,

$$
P\left\{X_{\underline{i}}<s, X_{\underline{j}}<t\right\}-P\left\{X_{\underline{i}}<s\right\} P\left\{X_{\underline{j}}<t\right\} \geq 0 .
$$

This concept was introduced by Lehmann [4]. In the following we will drop the assumption of positive dependence, but use the magnitude of the left hand sides in (1.1a) and (1.1b) as a measure of dependence.

For this reason we introduce the notion of asymptotic quadrant independence : A field $\left\{X_{j}: \underline{j} \in \mathbb{Z}_{+}^{d}\right\}$ is said to be pairwise asymptotically quadrant independent (AQQI), if there exists a sequence $\{q(\|\underline{n}\|): \underline{n} \in$ $\left.\mathbb{Z}_{+}^{d}\right\}$ with $q(\|\underline{n}\|) \longrightarrow 0$ as $\underline{n} \longrightarrow \infty$, such that for real $s, t$ and $\underline{i} \neq \underline{j}$, there holds

$$
\begin{aligned}
& \left|P\left\{X_{\underline{i}}>s, X_{\underline{j}}>t\right\}-P\left\{X_{\underline{i}}>s\right\} P\left\{X_{\underline{j}}>t\right\}\right| \\
\leq & q(\|\underline{j}-\underline{i}\|) \cdot \alpha_{\underline{i} \underline{j}}(s, t), \\
& \left|P\left\{X_{\underline{i}}<s, X_{\underline{j}}<t\right\}-P\left\{X_{\underline{i}}<s\right\} P\left\{X_{\underline{j}}<t\right\}\right| \\
\leq & q(\|\underline{j}-\underline{i}\|) \cdot \beta_{\underline{i} \underline{j}}(s, t),
\end{aligned}
$$

where $\alpha_{\underline{i} j}(s, t)$ and $\beta_{\underline{i} \underline{j}}(s, t)$ are nonnegative numbers which may depend on $\underline{i}, \underline{j}, s, t$. Since the left hand sides in (1.2a) and (1.2b) converge to zero as $|\bar{s}| \longrightarrow \infty$ and $|t| \longrightarrow \infty$, it seems natural to impose additional restrictions on $\alpha_{\underline{i j}}$ and $\beta_{\underline{\underline{\underline{j}}} \underline{\underline{j}}}$.

In the case of $d=1$ Birkel [2] introduced the concept of AQI and obtained the strong law of large numbers for AQI random variables. In this paper we extend the results obtained by Birkel [2] to the case of $d \geq 2$.

In Section 2 we consider some preliminary results which will be important role to prove the strong law of large numbers and we will derive conditions on pairwise AQI field $\left\{X_{\underline{j}}: \underline{j} \in \mathbb{Z}_{+}^{d}\right\}$ which quarantees that the finiteness of $\sum_{\underline{n} \in \mathbb{Z}_{+}^{d}}|\underline{n}|^{-1} q(\|\underline{n}\|)$ implies the strong law of large number in Section 3.

In the following statement, $\mathrm{C}$ stands for a constant whose value may vary from line to line. 


\section{Preliminaries}

The following theorems will be important roles to prove the strong laws of large for AQI field.

THEOREM 2.1. Let $\left\{X_{\underline{j}}: \underline{j} \in \mathbb{Z}_{+}^{d}\right\}$ be a field of nonnegative random variables with $E X_{\underline{j}}^{2}<\infty$ such that

(i) $\sup _{\underline{j} \in \mathbb{Z}_{+}^{d}} E X_{\underline{j}}<\infty$,
(ii) $\sum_{\underline{j} \geq \underline{1}} \sum_{|\underline{j}| \geq \mid \underline{\underline{i} \mid} \geq 1} \operatorname{Cov}^{+}\left(X_{\underline{i}}, X_{\underline{j}}\right) /|\underline{j}|^{2}<\infty$.

Then, as $\underline{n} \longrightarrow \infty$,

$$
|\underline{n}|^{-1} \sum_{\underline{1} \leq \underline{j} \leq \underline{n}}\left(X_{\underline{j}}-E X_{\underline{j}}\right) \longrightarrow 0 \text { a.s. }
$$

ProOF. It is enough to prove it for the 2-dimensional case. Let $a>1, b>1$ and $n_{\underline{k}}=\left(\left[a^{k_{1}}\right],\left[b^{k_{2}}\right]\right)$ for $\underline{k}=\left(k_{1}, k_{2}\right)$. By Chebyshev's inequality for every $\bar{\varepsilon}>0$

$$
\begin{aligned}
& \sum_{\underline{k} \geq \underline{1}} P\left\{\left|S_{n_{\underline{k}}}-E S_{n_{\underline{k}}}\right| \geq \varepsilon\left|n_{\underline{k}}\right|\right\} \\
& \leq C \sum_{\underline{k} \geq \underline{1}} \operatorname{Var}\left(S_{n_{\underline{k}}}\right) /\left|n_{\underline{k}}\right|^{2} \\
& \leq C \sum_{\underline{k} \geq \underline{1}} \sum_{\underline{1} \leq \underline{j} \leq n_{\underline{k}}} \sum_{\underline{1} \leq \underline{i} \leq n_{\underline{k}}} \operatorname{Cov}\left(X_{\underline{i}}, X_{\underline{j}}\right) /\left|n_{\underline{k}}\right|^{2}
\end{aligned}
$$

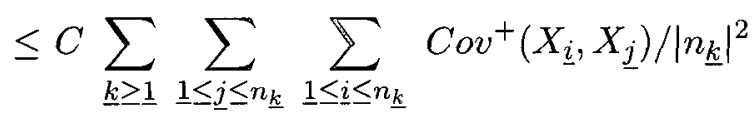

$$
\begin{aligned}
& =C \sum_{\underline{k} \geq \underline{1}} \frac{1}{\left|n_{\underline{k}}\right|^{2}} \sum_{\underline{1} \leq \underline{j} \leq n_{\underline{k}}} \sum_{1 \leq|\underline{i}| \leq|\underline{j}|} \operatorname{Cov}^{+}\left(X_{\underline{i}}, X_{\underline{j}}\right) \\
& =C \sum_{\underline{j} \geq \underline{1}} \sum_{1 \leq|\underline{i}| \leq|\underline{j}|} \operatorname{Cov}^{+}\left(X_{\underline{i}}, X_{\underline{j}}\right) \sum_{\left\{\underline{k}: n_{\underline{k}} \geq \underline{j}\right\}} \frac{1}{\left|n_{\underline{k}}\right|^{2}} \\
& \leq C \sum_{\underline{j} \geq \underline{1}} \sum_{1 \leq \underline{i}|\leq| \underline{j} \mid} \operatorname{Cov}^{+}\left(X_{\underline{i}}, X_{\underline{j}}\right) /|\underline{j}|^{2}<\infty \text {. }
\end{aligned}
$$


The last inequality of (2.2) follows from the following : First note that

$$
\sum_{\left\{\underline{k}: n_{\underline{k} \geq \underline{j}}\right\}} \frac{1}{\left|n_{\underline{k}}\right|^{2}}=\sum_{\underline{k} \geq \underline{k_{0}}} \frac{1}{\left|n_{\underline{k}}\right|^{2}}
$$

where $\underline{k_{0}}=\min \left\{\underline{k}: n_{\underline{k}} \geq \underline{j}\right\}$. Then the right-hand side of (2.3) yields

$$
\begin{aligned}
& \sum_{\underline{k} \geq \underline{k_{0}}} \frac{1}{\left|n_{\underline{k}}\right|^{2}} \leq C \sum_{\underline{k} \geq \underline{k_{0}}} \frac{1}{a^{2 k_{1}} b^{2 k_{2}}} \\
& =C \sum_{k_{2} \geq k_{0}^{\prime \prime}} \sum_{k_{1} \geq k_{0}^{\prime}} \frac{1}{a^{2 k_{1}} b^{2 k_{2}}} \\
& \leq \frac{D}{a^{2 k_{0}^{\prime} b^{2 k_{0}^{\prime \prime}}}} \\
& \leq \frac{D}{\left|{\underline{n_{\underline{k}}}}^{2}\right|^{2}} \leq \frac{E}{|\underline{j}|^{2}}
\end{aligned}
$$

where $\underline{k_{0}}=\left(k_{0}^{\prime}, k_{0}^{\prime \prime}\right)$ and $C, D$ and $E$ are some positive constants. Thus by the Borel-Cantelli lemma it follows from (2.2) that

$$
\left(S_{n_{\underline{k}}}-E S_{n_{\underline{k}}}\right) /\left|n_{\underline{k}}\right| \rightarrow 0 \text { a.s. }
$$

Now given $\underline{k}=\left(k_{1}, k_{2}\right)$, positive integers $k_{1}, k_{2}$ for $n_{\underline{k}} \leq \underline{n} \leq n_{\underline{k}+\underline{1}}$ we have

$$
\left|\frac{S_{\underline{n}}-E S_{\underline{n}}}{|\underline{n}|}\right| \leq\left|\frac{S_{n_{\underline{k}+1}}-E S_{n_{\underline{k}+1}}}{\left|n_{\underline{k}}+\underline{1}\right|}\right| \frac{\left|n_{\underline{k}+1}\right|}{\left|n_{\underline{k}}\right|}+\frac{E S_{n_{\underline{\underline{k}}+\underline{1}}}-E S_{n_{\underline{k}}}}{\left|n_{\underline{k}}\right|}
$$

by the monotonicity of $S_{\underline{n}}$. Let $a>1, b>1$ and for each $n_{\underline{k}}=\left(n_{k_{1}}, n_{k_{2}}\right)$ set $n_{k_{1}}=\left[a^{k_{1}}\right], n_{k_{2}}=\left[b^{k_{2}}\right]$. Then from $(i),(2.4)$ and (2.5) one can easily verify that

$$
\lim _{\underline{n} \geq \underline{1}} \sup \left(\left|S_{\underline{n}}-E S_{\underline{n}}\right| /|\underline{n}|\right) \leq \sup _{\underline{j} \geq \underline{1}} E X_{\underline{j}}(a b-1),
$$

for every $a>1$ and $b>1$ which concludes the proof since both $a$ and $b$ may arbitrary close to 1 .

ThEOREM 2.2. Let $\left\{X_{\underline{j}}: \underline{j} \in \mathbb{Z}_{+}^{d}\right\}$ be a field of nonnegative $d$ dimensional random variable such that

(i) $\sup E X_{j}<\infty$, $\underline{j} \in \mathbb{Z}_{+}^{d}$

(ii) $\sum_{\underline{j} \geq \underline{1}} P\left(X_{\underline{j}}>|\underline{j}|\right)<\infty,|\underline{n}|^{-1} \sum_{\underline{1} \leq \underline{j} \leq \underline{n}} E X_{\underline{j}} 1_{\left\{X_{\underline{j}} \geq|\underline{j}|\right\}} \longrightarrow 0$ as $\underline{n} \longrightarrow \infty$, 
(iii) $\sum_{\underline{j} \geq \underline{1}} \sum_{|\underline{j}| \geq|\underline{i}| \geq 1}|\underline{j}|^{-2} \operatorname{Cov}^{+}\left(X_{\underline{i}} 1_{\left\{X_{\underline{i}} \leq|\underline{i}|\right\}}, X_{\underline{j}} 1_{\left\{X_{\underline{j}} \leq|\underline{j}|\right\}}\right)<\infty$.

Then, as $\underline{n} \longrightarrow \infty$,

$$
|\underline{n}|^{-1} \sum_{\underline{1} \leq \underline{j} \leq \underline{n}}\left(X_{\underline{j}}-E X_{\underline{j}}\right) \longrightarrow 0 \text { a.s.. }
$$

Proof. For $\underline{j} \in \mathbb{Z}_{+}^{d}$ let $Y_{\underline{j}}=X_{\underline{j}} 1_{\left\{X_{\underline{j}} \leq|\underline{j}|\right\}}$. Clearly, the random field $\left\{Y_{j}: \underline{j} \in \mathbb{Z}_{+}^{d}\right\}$ satisfies the assumptions of Theorem 2.1. Hence as $\underline{n} \stackrel{-}{\longrightarrow} \infty$,

$$
|\underline{n}|^{-1} \sum_{\underline{1} \leq \underline{j} \leq \underline{n}}\left(Y_{j}-E Y_{\underline{j}}\right) \longrightarrow 0 \text { a.s. }
$$

Assumption (ii) of Theorem 2.2 takes care of the difference between $\sum_{1 \leq \underline{j} \leq \underline{n}} Y_{\underline{j}}$ and $\sum_{1 \leq \underline{j} \leq \underline{n}} X_{\underline{j}}$, and $\sum_{\underline{1} \leq \underline{j} \leq \underline{n}} E Y_{\underline{j}}$ and $\sum_{\underline{1} \leq \underline{j} \leq \underline{n}} E X_{\underline{j}}$, which is sufficient to get the desired result.

LEMMA 2.3. (Newman [5]) Let $X_{1}, X_{2}$ be random variables with $E X_{j}^{2}<\infty$ for $j=\underline{1}, \underline{2}$. Then

$$
\begin{aligned}
& \operatorname{Cov}\left(X_{\underline{1}}, X_{\underline{2}}\right) \\
= & \int_{-\infty}^{\infty} \int_{-\infty}^{\infty}\left(P\left\{X_{\underline{1}}>s, X_{\underline{2}}>t\right\}-P\left\{X_{\underline{1}}>s\right\} P\left\{X_{\underline{2}}>t\right\}\right) d s d t
\end{aligned}
$$

Lemma 2.4. (Birkel [2]) Let $X$ be a random variable with $E X^{2}<\infty$. Then
(i) $\operatorname{Var}\left(X^{+}\right) \leq \operatorname{Var}(X)$
(ii) $\operatorname{Var}\left(X^{-}\right) \leq \operatorname{Var}(X)$.

\section{Main results}

In this section we extend some results in Birkel [2] to the $d$-dimensional case.

THEOREM 3.1. Let $\left\{X_{j}: \underline{j} \in \mathbb{Z}_{+}^{d}\right\}$ be an $A Q I$ field of uniformly bounded random variables with $E X_{\underline{j}}=0$ such that

$$
\begin{aligned}
\alpha_{\underline{i}, \underline{j}}(s, t) & \leq C_{\alpha}<\infty, \\
\beta_{\underline{i}, \underline{j}}(s, t) & \leq C_{\beta}<\infty .
\end{aligned}
$$


If $\{q(\|\underline{n}\|): \underline{n} \geq \underline{1}\}$ satisfies $\sum_{\underline{n} \geq \underline{1}}|\underline{n}|^{-1} q(\|\underline{n}\|)<\infty$, then $\left\{X_{\underline{j}}: \underline{j} \in \mathbb{Z}_{+}^{d}\right\}$ fulfills the strong law of large numbers.

Proof. Let $M<\infty$ be such that $\left|X_{\underline{j}}\right| \leq M$ for $\underline{j} \in \mathbb{Z}_{+}^{d}$. We will verify that

$$
|\underline{n}|^{-1} \sum_{\underline{1} \leq \underline{j} \leq \underline{n}}\left(X_{\underline{j}}^{+}-E X_{\underline{j}}^{+}\right) \longrightarrow 0 \text { a.s. }
$$

and

$$
|\underline{n}|^{-1} \sum_{\underline{1} \leq \underline{j} \leq \underline{n}}\left(X_{\underline{j}}^{-}-E X_{\underline{j}}^{-}\right) \longrightarrow 0 a . s . .
$$

As $\sup E X_{\underline{j}}^{+} \leq M<\infty$, by using Theorem 2.1 it suffices to prove $\underline{j} \in \mathbb{Z}_{+}^{d}$

$$
\sum_{\underline{j} \geq \underline{1}} \sum_{|\underline{j}| \geq|\underline{j}| \geq|\underline{1}|}|\underline{j}|^{-2} \operatorname{Cov}^{+}\left(X_{\underline{i}}^{+}, X_{\underline{j}}^{+}\right)<\infty .
$$

Applying Lemma 2.3 and the pairwise AQI property (1.2a), we obtain for $\underline{i} \neq \underline{j}$,

$$
\begin{aligned}
& \operatorname{Cov}^{+}\left(X_{\underline{i}}^{+}, X_{\underline{j}}^{+}\right) \\
\leq & \left|\int_{0}^{M} \int_{0}^{M}\left(P\left\{X_{\underline{i}}^{+}>s, X_{\underline{j}}^{+}>t\right\}-P\left\{X_{\underline{i}}^{+}>s\right\} P\left\{X_{\underline{j}}^{+}>t\right\}\right) d s d t\right| \\
\leq & \int_{0}^{M} \int_{0}^{M}\left|P\left\{X_{\underline{i}}^{+}>s, X_{\underline{j}}^{+}>t\right\}-P\left\{X_{\underline{i}}^{+}>s\right\} P\left\{X_{\underline{j}}^{+}>t\right\}\right| d s d t \\
\leq & q(\|\underline{j}-\underline{i}\|) \int_{0}^{M} \int_{0}^{M} \alpha_{\underline{i}, \underline{j}}(s, t) d s d t \\
\leq & C_{\alpha} \cdot M^{2} q(\|\underline{j}-\underline{i}\|) .
\end{aligned}
$$


Hence

$$
\begin{aligned}
& \sum_{\underline{j} \geq \underline{1}} \sum_{\underline{1} \leq|\underline{i}| \leq|\underline{j}|}|\underline{j}|^{-2} \operatorname{cov}^{+}\left(X_{\underline{i}}^{+}, X_{\underline{j}}^{+}\right) \\
\leq & C_{\alpha} \sum_{\underline{j} \geq \underline{1}} \sum_{1 \leq|\underline{i}| \leq|\underline{j}|}|\underline{j}|^{-2} M^{2} q(\|\underline{j}-\underline{i}\|)+\sum_{\underline{j} \geq \underline{1}}|\underline{j}|^{-2} \operatorname{Var}\left(X_{j}^{+}\right) \\
\leq & C_{\alpha} M^{2} \sum_{|\underline{n}| \geq \underline{1}} q(\|\underline{n}\|) \sum_{|\underline{l}| \geq|\underline{n}|+1}(\|\underline{l}\|)^{-2}+M^{2} \sum_{\underline{j} \geq \underline{1}}|\underline{j}|^{-2} \\
\leq & C_{1} M^{2} \sum_{|\underline{n}| \geq \underline{1}}|\underline{n}|^{-1} q(\|\underline{n}\|)+C_{2} M^{2}<\infty .
\end{aligned}
$$

This proves (3.3). Thus, by Theorem 2.1 we have as $\underline{n} \longrightarrow \infty$,

$$
|\underline{n}|^{-1} \sum_{\underline{1} \leq \underline{j} \leq \underline{n}}\left|X_{\underline{j}}^{+}-E X_{\underline{j}}^{+}\right| \longrightarrow 0 \text { a.s.. }
$$

Using that for $s, t \geq 0$,

$$
\begin{gathered}
P\left\{X_{\underline{i}}^{-}>s, X_{\underline{j}}^{-}>t\right\}-P\left\{X_{\underline{i}}^{-}>s\right\} P\left\{X_{\underline{j}}^{-}>t\right\} \\
=P\left\{X_{\underline{i}}^{-}<-s, X_{\underline{j}}^{-}<-t\right\}-P\left\{X_{\underline{i}}^{-}<-s\right\} P\left\{X_{\underline{j}}^{-}<-t\right\}
\end{gathered}
$$

and applying the pairwise AQI property $(1.2 \mathrm{~b}),(3.2)$ is verified in the same way. Since $X_{\underline{j}}^{+}-X_{\underline{j}}^{-}=X_{\underline{j}}, E X_{\underline{j}}^{+}-E X_{\underline{j}}^{-}=0$, this gives us the desired result.

If the random variables are not uniformly bounded, $\alpha_{\underline{i}, \underline{j}}$ and $\beta_{\underline{i}, \underline{j}}$ have to satisfy additional assumptions.

THEOREM 3.2. Let $\left\{X_{j}: \underline{j} \in \mathbb{Z}_{+}^{d}\right\}$ be a field of AQI d-dimensional random variables with $E X_{\underline{j}}=0$ such that

$$
\sup _{\underline{i} \neq \underline{j}} \int_{0}^{\infty} \int_{0}^{\infty} \alpha_{\underline{i}, \underline{j}}(s, t) d s d t<\infty, \sup _{\underline{i \neq} \underline{j}} \int_{0}^{\infty} \int_{0}^{\infty} \beta_{\underline{i}, \underline{j}}(-s,-t) d s d t<\infty .
$$

Assume

$$
\begin{aligned}
& \text { (i) } \sup _{\underline{j} \in \mathbb{Z}_{+}^{d}} E\left|X_{\underline{j}}\right|<\infty, \\
& \text { (ii) } \quad E X_{\underline{j}}^{2}<\infty, \sum_{\underline{j} \geq \underline{\underline{1}}}|\underline{j}|^{-2} \operatorname{Var}\left(X_{\underline{j}}\right)<\infty .
\end{aligned}
$$

If $\{q(\|\underline{n}\|): \underline{n} \geq \underline{1}\}$ satisfies $\sum_{|\underline{n}| \geq 1}|\underline{n}|^{-1} q(\|\underline{n}\|)<\infty$ then $\left\{X_{\underline{j}}: \underline{j} \in \mathbb{Z}_{+}^{d}\right\}$ fulfills the strong law of large numbers. 
Proof. We proceed as in the proof of Theorem 3.1. Using the integrability of $\alpha_{\underline{i}, \underline{j}}$, we get for $\underline{i} \neq \underline{j}$

$$
\begin{aligned}
\operatorname{Cov}^{+}\left(X_{\underline{i}}^{+}, X_{\underline{j}}^{+}\right) & \leq q(\|\underline{j}-\underline{i}\|) \int_{0}^{\infty} \int_{0}^{\infty} \alpha_{\underline{i}, \underline{j}}(s, t) d s d t \\
& \leq C_{\alpha} \cdot q(\|\underline{j}-\underline{i}\|),
\end{aligned}
$$

and hence,

$$
\begin{aligned}
& \sum_{\underline{j} \geq \underline{1}} \sum_{|\underline{j}| \geq|\underline{i}| \geq|\underline{1}|}|\underline{j}|^{-2} \operatorname{Cov}^{+}\left(X_{\underline{i}}^{+}, X_{\underline{j}}^{+}\right) \\
\leq & C \sum_{|\underline{n}| \geq \underline{1}}|\underline{n}|^{-1} q(\|\underline{n}\|)+\sum_{\underline{j} \geq \underline{1}}|\underline{j}|^{-2} \operatorname{Var}\left(X_{\underline{j}}\right)<\infty,
\end{aligned}
$$

by Lemma 2.4 and assumption (ii). Now the proof of Theorem 3.2 follows along the lines of the proof of Theorem 3.1.

REMARK. The strong law of large numbers, resulting from Theorem 3.2 , shows the possibility that Theorem 1 of Birkel [1] and Etemadi's strong law of large numbers ([3], Corollary 1 ) can be extended to the case of $d \geq 2$.

\section{References}

[1] T. Birkel, A note on the strong law of large numbers for positively dependent random variables, Statist. Probab. Lett. 7 (1989), 17-20.

[2] _ Laws of large numbers under dependence assumptions, Statist. Probab. Lett. 14 (1992), 355-362.

[3] N. Etemadi, On the strong laws of large numbers for nonnegative random variables, J. Multivariate Anal. 13 (1983), 187-193.

[4] E. L. Lehmann, Some concepts of dependence, Ann. Math. Statist. 37 (1966), $1137-1153$.

[5] C. M. Newman, Asymptotic independence and limit theorems for positively and negatively dependent random variables. in : Y.L. Tong, ed., Inequalities in Statistics and Probability, IMS, Hayward CA, 1984, 127-140.

\section{Mi-Hwa Ko}

Statistical Research Center for Complex Systems

Seoul National University

Seoul 151-742, Korea

E-mail: kmh@srccs.snu.ac.kr 
Tae-Sung Kim

Department of Mathematics and Institute of Basic Science

WonKwang University

Jeonbuk 570-749, Korea

E-mail: starkim@wonkwang.ac.kr

Hyun-Chull Kim

Division of Computer and Information Science

Daebul University

Chonnam 526-702, Korea

E-mail: kimhc@mail.daebul.ac.kr 
